\title{
Mixtures of Charged Colloid and Neutral Polymer: Influence of Electrostatic Interactions on Demixing and Interfacial Tension
}

\author{
Alan R. Denton* \\ Department of Physics, North Dakota State University, Fargo, ND, 58105-5566 \\ Matthias Schmidt ${ }^{\dagger}$ \\ Institut für Theoretische Physik II, Heinrich-Heine-Universität Düsseldorf, \\ Universitätsstraße 1, D-40225 Düsseldorf, Germany
}

(Dated: September 13, 2018)

\begin{abstract}
The equilibrium phase behavior of a binary mixture of charged colloids and neutral, nonadsorbing polymers is studied within free-volume theory. A model mixture of charged hard-sphere macroions and ideal, coarse-grained, effective-sphere polymers is mapped first onto a binary hardsphere mixture with non-additive diameters and then onto an effective Asakura-Oosawa model [S. Asakura and F. Oosawa, J. Chem. Phys. 22, 1255 (1954)]. The effective model is defined by a single dimensionless parameter - the ratio of the polymer diameter to the effective colloid diameter. For high salt-to-counterion concentration ratios, a free-volume approximation for the free energy is used to compute the fluid phase diagram, which describes demixing into colloid-rich (liquid) and colloid-poor (vapor) phases. Increasing the range of electrostatic interactions shifts the demixing binodal toward higher polymer concentration, stabilizing the mixture. The enhanced stability is attributed to a weakening of polymer depletion-induced attraction between electrostatically repelling macroions. Comparison with predictions of density-functional theory reveals a corresponding increase in the liquid-vapor interfacial tension. The predicted trends in phase stability are consistent with observed behavior of protein-polysaccharide mixtures in food colloids.

PACS numbers: 61.20.Gy, 64.70.Ja, 82.70.Dd, 05.20.Jj, 05.70.-a
\end{abstract}

\footnotetext{
* Electronic address: alan.denton@ndsu.edu

$\dagger$ Electronic address: mschmidt@thphy.uni-duesseldorf.de
} 


\section{INTRODUCTION}

Mixtures of colloidal particles and free (non-adsorbing) polymer coils dispersed in a solvent are among the most intensively studied soft matter systems 1 1.2.3.4.5.6. The conceptual analogy between colloids and atoms, similarities in thermodynamic phase behavior between colloidal suspensions and atomic systems, and the relative ease of tuning polymer-induced effective colloidal interactions, make colloid-polymer mixtures valuable model systems for probing connections between microscopic interparticle interactions and macroscopic properties in a variety of materials. The observed phases, distinguished by composition and structural order, include most of the equilibrium phases familiar in simple molecular systems, e.g., vapor, liquid, crystal, as well as nonequilibrium states, such as glasses and gels $\stackrel{1.7}{ }$. Complementing their fundamental importance, colloid-polymer mixtures have diverse industrial applications, e.g., to coatings, petroleum products, pharmaceuticals, and many foods, where polymer additives are used to control phase stability and rheological properties.

Addition of free polymer can substantially modify effective interactions between colloidal particles through the mechanism of entropic depletion. When two colloidal surfaces approach to a separation closer than the typical diameter of a polymer coil, the entropic cost to the coil of distorting its average spherical conformation tends to exclude the polymer. The resulting depletion of polymer from the space between the colloids creates an imbalance in polymer osmotic pressure that can induce effective attractions between colloids. Attractions of sufficient range and strength, depending on the relative size and concentration of polymer, can drive bulk demixing into colloid-rich (liquid) and colloid-poor (vapor) phases.

The first, and conceptually simplest, statistical mechanical model to qualitatively describe the polymer depletion mechanism and the associated phenomenon of depletion-driven demixing was the model of Asakura and Oosawa ${ }^{8}$, developed independently by Vrij ${ }^{9}$. The Asakura-Oosawa (AO) model regards the colloidal particles as hard spheres and the polymer coils as effective ideal spheres - mutually noninteracting, but interacting with the colloids as hard spheres. Neglect of polymer-polymer interactions can be reasonably justified for polymers in a theta solvent. The phase diagram corresponding to the AO model has been determined by a variety of methods, including thermodynamic perturbation theory $\underline{10}^{10}$, freevolume theory ${ }^{11.12}$, density-functional theory ${ }^{13}$, and Monte Carlo simulation ${ }^{14.15 .16}$. Recent 
effort has also been devoted to calculating the liquid-vapor interfacial tension of phaseseparated colloid-polymer mixtures $\frac{17.18 .1920 .21 .22 .23 .24 .25 .26}{}$.

Although certain topologically constrained polymers (e.g., stars and microgels) are close to spherical in shape, linear-chain polymers are random walks whose shapes are spherical only on average. To explore the significance of nonspherical conformations, more explicit segmented-chain polymer models have been studied via Monte Carlo simulation²7,28,29 and integral-equation theory ${ }^{30}$. Effective polymer-polymer interactions have been modeled by combining Monte Carlo and integral-equation methods within a "polymers as soft colloids" framework ${ }^{29}$. In recent work, we examined fluid-fluid demixing within several variations of the classic AO model, incorporating a third component (hard needles) ${ }^{31}$, polymer-solvent interactions ${ }^{32}$, polymer-polymer interactions $s^{33}$, and colloid-induced polymer compression ${ }^{34}$. The latter study incorporated intrinsic polydispersity in the polymer radius of gyration. Influences on phase behavior of colloid polydispersity ${ }^{35}$ and of polymer chain length polydispersity $\underline{36,37}$ have also been studied theoretically.

While neutral colloid-polymer mixtures have been widely investigated, much less attention has been devoted to mixtures of charged species. In the case of charged colloids or charged polymers (polyelectrolytes), electrostatic repulsions between macroions, screened by counterions and salt ions in solution, compete with depletion-induced attractions and can modify phase behavior. In pioneering experiments ${ }^{38.39}$, phase separation was observed in mixtures of charged colloids and neutral polymers. Gast et al $\stackrel{39}{\underline{3}}$ interpreted their observations by applying thermodynamic perturbation theory to an effective one-component model, incorporating electrostatic repulsion and depletion-induced attraction into an effective pair potential between colloids. Subsequent measurements of force profiles in mixtures of charge-stabilized colloidal oil-in-water emulsion droplets and ionic surfactant micelles $\underline{40}$ and in mixtures of colloidal particles and charged macromolecules ${ }^{41}$ directly demonstrated the potential for electrostatic interactions to modify depletion forces. More recently, liquidvapor separation and gelation were observed in mixtures of charged colloids and ionic wormlike micelles $\underline{42}$ and depletion potentials induced by charged rods were measured in colloidal rod-sphere mixtures ${ }^{43}$. In a recent theoretical study, liquid-state integral-equation methods were used to model the structure and phase behavior of mixtures of charged colloids and polyelectrolytes $\stackrel{44}{ }$. 
The purpose of the present paper is to propose, for a simple model of a charged-colloid - neutral-polymer mixture, an alternative theoretical approach that, in contrast to Ref. 39, treats the two components on an equal footing. The theory is based on mapping the binary mixture onto an effective AO model, governed only by excluded volume interactions, and applying free-volume theory ${ }^{11}$ and classical density-functional theory ${ }^{13}$. Within this conceptual approach, we explore the qualitative influence of colloidal charge on demixing and find that increasing the range of electrostatic interactions can significantly stabilize the mixture against demixing and correspondingly increase the liquid-vapor interfacial tension.

The remainder of the paper is organized as follows. The model system is defined in Sec. II] The mapping onto the effective AO model and the free-volume theory are described in Sec. [II] Results for the fluid-fluid demixing phase diagram and interfacial tension are presented and discussed in Sec. IV] Finally, Sec. $\nabla$ closes with a summary and conclusions.

\section{MODEL}

The system of interest comprises charged colloidal particles, their dissociated counterions, and free polymer coils, all dispersed in an electrolyte solvent. The multi-component mixture of macroions, microions (counterions and salt ions), polymers, and solvent molecules spans a range of length and time scales, presenting severe challenges to explicit modeling approaches. To reduce the system to a tractable model, we make several simplifying, yet realistic, assumptions. (1) The colloids are modeled as monodisperse charged hard spheres - a close approximation to many real synthetic suspensions. (2) The microions are modeled as monovalent point charges and subsumed into effective electrostatic interactions between macroions, as described below. (3) The polymer coils are represented, as in the coarsegrained AO model, as effective spheres. Nonspherical conformations, disfavored by lower conformational entropy, can be reasonably neglected when the polymer coils are comparable or smaller in size than the colloids (colloid limit). (4) Finally, the solvent is treated as a di-

electric continuum, characterized by a dielectric constant (primitive model of electrolytes ${ }^{45}$ ). An experimental system that closely resembles our model system would be charge-stabilized synthetic polystyrene or silica microspheres dispersed in an aqueous electrolyte together with a non-adsorbing, nonionic, water-soluble polymer, such as hydroxyethylcellulose (HEC) $)^{38.39}$ 
or polyethylene oxide (PEO).

Interactions between colloidal particles include steric and electrostatic repulsions and van der Waals attractions. Here we assume hard-sphere steric interactions and ignore van der Waals interactions, which is valid for particles index-matched to the solvent. Electrostatic interactions between colloidal macroions result from bare Coulomb repulsion and screening by surrounding microions. The classic theory of Derjaguin, Landau, Verwey, and Overbeek $(\mathrm{DLVO})^{46}$ predicts that, in a dilute suspension, two colloidal macroions at center-to-center separation $r$ interact via a pair potential of screened-Coulomb (Yukawa) form:

$$
v_{c c}(r)= \begin{cases}\infty, & r<2 R_{c} \\ \frac{Z^{2} e^{2}}{\epsilon}\left(\frac{e^{\kappa R_{c}}}{1+\kappa R_{c}}\right)^{2} \frac{e^{-\kappa r}}{r}, & r \geq 2 R_{c}\end{cases}
$$

where $Z$ is the effective macroion valence, $e$ the proton charge, $\epsilon$ the solvent dielectric constant, $\kappa$ the Debye screening constant (inverse screening length), and $R_{c}$ the colloid radius.

More recent statistical mechanical approaches proceed by formally integrating out from the partition function the microion degrees of freedom to map the macroion-microion mixture onto an effective one-component system governed by effective interactions $47,48,49,50,51,52$, determined by the distribution of microions around the macroions. The microion distribution depends on the response - in general nonlinear - of the microions to the macroion charge density. For sufficiently weakly charged macroions, the microion response can be approximated as linear. A further neglect of microion correlations, which is reasonable for monovalent counterions, then recovers the DLVO form of effective pair potential [Eq. (10)] with a density-dependent screening constant of the form

$$
\kappa=\sqrt{\frac{4 \pi\left(Z \rho_{c}+2 \rho_{s}\right) e^{2}}{\epsilon k_{\mathrm{B}} T}},
$$

where $\rho_{c}$ and $\rho_{s}$ are, respectively, the number densities of colloidal macroions and salt ion pairs, $k_{\mathrm{B}}$ is Boltzmann's constant and $T$ is the absolute temperature. The nonzero size of the microions may modify the microion distribution and thus screening effect, but should not qualitatively change the general repulsive form of the effective pair potential assumed here. In practice, since most water-soluble polymers are less polarizable than water, $\epsilon$ may 
be best interpreted as an effective dielectric constant of the polymer-solvent mixture and $\kappa$ as an effective screening constant. For the purposes of this paper, the essential point is that the range of electrostatic repulsion, governed by $\kappa$, can be widely tuned by adjusting the ionic strength (salt concentration) of the electrolyte solvent.

As a natural byproduct of the one-component mapping, the total free energy contains, in addition to a pair-interaction contribution, a one-body volume energy $y^{47.48,49,50,51.52} E$, given by

$\frac{E}{V k_{\mathrm{B}} T}=\left(Z \rho_{c}+\rho_{s}\right)\left\{\ln \left[\left(Z \rho_{c}+\rho_{s}\right) \Lambda^{3}\right]-1\right\}+\rho_{s}\left\{\ln \left(\rho_{s} \Lambda^{3}\right)-1\right\}-\frac{Z^{2} \rho_{c} \kappa \lambda_{\mathrm{B}}}{2\left(1+\kappa R_{c}\right)}-\frac{Z^{2} \rho_{c}^{2}}{2\left(Z \rho_{c}+2 \rho_{s}\right)}$,

where $V$ is the total volume of the system, $\Lambda$ is the thermal wavelength of the microions and $\lambda_{\mathrm{B}}=e^{2} /\left(\epsilon k_{\mathrm{B}} T\right)$ is the Bjerrum length. In a straightforward physical interpretation, the first two terms in Eq. (3) account for the microion entropy, the third term is the interaction of a macroion with its own cloud of counterions, and the final term results from charge neutrality. Because the volume energy depends nontrivially on colloid density, it must, in general, be included in the free energy. At relatively high salt concentrations $\left(\rho_{s} \gg Z \rho_{c}\right)$, however, the counterions are dominated by salt ions and the volume energy can be neglected. We restrict our considerations to this parameter regime.

Within the coarse-grained spherical polymer model, colloid-polymer interactions are described by a simple excluded-volume pair potential:

$$
v_{c p}(r)= \begin{cases}\infty, & r<R_{c}+R_{p} \\ 0, & r \geq R_{c}+R_{p}\end{cases}
$$

where $r$ is now the macroion-polymer center-to-center distance and $R_{p}$ is the polymer radius of gyration. For simplicity, we assume the solvent to be near its theta temperature for the polymer ${ }^{53}$, allowing the polymer to be reasonably modeled as ideal (mutually noninteracting), i.e., $v_{p p}(r)=0$, for all $r$. In practice, the strength of polymer-polymer interactions will depend on the properties of specific polymers and solvents.

Within the above assumptions, the model system is now completely characterized by the colloid-colloid interaction [Eqs. (11) and (2)] and the polymer-to-colloid size ratio $q=R_{p} / R_{c}$. The thermodynamic states of the system are specified by the bulk number densities $\rho_{i}$, or equivalently the volume fractions, $\eta_{i}=(4 \pi / 3) \rho_{i} R_{i}^{3}$, of species $i=c, p$. 


\section{THEORY}

\section{A. Mapping onto the Asakura-Oosawa Model}

To explore thermodynamic properties of the model colloid-polymer mixture, we seek an approximation for the free energy of the system. We proceed by first constructing a mapping onto a simpler model system. A similar approach has been developed recently by Tuinier ${ }^{55}$. Assuming the screened-Coulomb effective pair potential between colloids [Eq. (11)] to be relatively steeply repulsive $\left(\kappa R_{c}>1\right)$, the corresponding contribution to the free energy may be mapped, with reasonable accuracy, onto the free energy of an effective hard-sphere system ${ }^{45,56}$ interacting via an effective hard-sphere pair potential,

$$
v_{c c}^{\prime}(r)= \begin{cases}\infty, & r<2 R_{c}^{\prime} \\ 0, & r \geq 2 R_{c}^{\prime}\end{cases}
$$

where $R_{c}^{\prime}$ is the effective hard-sphere colloid radius. A reasonable first estimate of the effective radius is obtained from a simple thermal criterion,

$$
v_{c c}\left(r=2 R_{c}^{\prime}\right)=\frac{Z^{2} e^{2}}{\epsilon}\left(\frac{e^{\kappa R_{c}}}{1+\kappa R_{c}}\right)^{2} \frac{e^{-2 \kappa R_{c}^{\prime}}}{2 R_{c}^{\prime}}=k_{\mathrm{B}} T,
$$

according to which colloids tend not to approach closer than a distance at which their interaction energy is comparable to the typical thermal energy.

Assuming high salt-to-counterion concentration ratios $\left(\rho_{s} \gg Z \rho_{c}\right)$, the screening constant $\kappa$, and thus $v_{c c}(r)$ and $R_{c}^{\prime}$, are practically independent of density. In this same limit, the volume energy [Eq. (3)] contributes to the free energy density a term that is only linear in colloid density and therefore irrelevant for phase behavior. To demonstrate that in this high-salt-concentration regime the effective colloid radius may still significantly exceed the bare radius, consider the typical example of particles of bare radius $R_{c}=50 \mathrm{~nm}$ and effective valence $Z=500$ suspended at volume fraction $\eta_{c}=0.01$ in a $1 \mathrm{mM}$ aqueous salt solution at room temperature. In this case, $\rho_{s} \gg Z \rho_{c}=0.016 \mathrm{mM}$, yet the effective colloid radius is estimated [from Eq. (6)] to be $R_{c}^{\prime} \simeq 67 \mathrm{~nm}$, i.e., about $30 \%$ greater than the bare radius, a difference that also well exceeds typical colloid polydispersities.

The original system has thus far been mapped onto an effective binary hard-sphere mixture, governed by the pair interactions of Eqs. (4) and (5). As a measure of the relative range 
of electrostatic and excluded-volume repulsions, it is convenient to define the electrostatic colloid size ratio, $\xi=R_{c}^{\prime} / R_{c} \geq 1$, as the ratio of the effective and bare colloid radii. The model system is now competely specified by two dimensionless parameters, $\xi$ and $q$. Since the polymer species is ideal, this model resembles the AO model, with the colloid diameter merely rescaled. However, because colloids and polymers repel at a range of $\left(R_{c}+R_{p}\right)$, rather than $\left(R_{c}^{\prime}+R_{p}\right)$, the hard-sphere diameters are no longer additive and the rescaling is nontrivial. Nevertheless, as Fig. 1 illustrates, this non-additive mixture can be further mapped exactly onto an effective additive AO model by introducing a fictitious effective polymer radius $R_{p}^{\prime}$, defined via

$$
R_{c}^{\prime}+R_{p}^{\prime}=R_{c}+R_{p}
$$

so as to yield the correct colloid-polymer excluded-volume interaction [Eq. (4)].

The final effective AO model is characterized by a single dimensionless parameter, namely the effective polymer-to-colloid size ratio

$$
q^{\prime}=R_{p}^{\prime} / R_{c}^{\prime}=(q-\xi+1) / \xi, \quad \text { for } \xi \leq 1+q
$$

As shown in Fig. 2, the rescaled polymer-to-colloid size ratio is always smaller than the true ratio $\left(q^{\prime}<q\right)$ and with increasing electrostatic colloid size ratio, $q^{\prime}$ decreases, i.e., the polymers grow effectively smaller relative to the colloids. For $\xi>1+q$, there is no polymer depletion, and so then $q^{\prime}=0$. The thermodynamic states are in turn specified by effective volume fractions

$$
\eta_{c}^{\prime}=\frac{4 \pi}{3} \rho_{c} R_{c}^{\prime 3}=\xi^{3} \eta_{c}
$$

and

$$
\eta_{p}^{\prime}=\frac{4 \pi}{3} \rho_{p} R_{p}^{\prime 3}=[1-(\xi-1) / q]^{3} \eta_{p}
$$

where we have used the relation $R_{p}^{\prime} / R_{p}=1-(\xi-1) / q<1$, obtained from Eq. (17).

\section{B. Free-Volume Theory}

Having mapped the original mixture of charged colloids and neutral polymers onto an effective AO model, we now consider the Helmholtz free energy, from which all equilibrium 
thermodynamic properties may be determined. The total free energy, $F=F_{\text {id }}+F_{\text {ex }}$, separates conveniently into an ideal-gas term $F_{\text {id }}$, which is independent of interactions, and an excess term $F_{\text {ex }}$, which depends entirely on interactions. For a homogeneous fluid, the ideal-gas free energy density is given exactly by

$$
\beta F_{\mathrm{id}} / V=\rho_{c}\left[\ln \left(\rho_{c} \Lambda_{c}^{3}\right)-1\right]+\rho_{p}\left[\ln \left(\rho_{p} \Lambda_{p}^{3}\right)-1\right]
$$

where $\beta=1 / k_{\mathrm{B}} T$ and $\Lambda_{c}$ and $\Lambda_{p}$ are the thermal wavelengths of the colloid and polymer species. For the excess free energy, we apply the mean-field free-volume theory of Lekkerkerker et $a l . \stackrel{11}{ }$, which predicts fluid-fluid phase separation for the original AO model in good agreement with simulation ${ }^{14}$ 15.23.24. Within this approach, the fluid excess free energy density is approximated by

$$
\beta F_{\mathrm{ex}} / V=\beta \phi_{\mathrm{HS}}\left(\eta_{c}^{\prime}\right)-\rho_{p} \ln \alpha\left(\eta_{c}^{\prime}, q^{\prime}\right)
$$

where $\phi_{\mathrm{HS}}\left(\eta_{c}^{\prime}\right)$ is the excess free energy density of the effective one-component hard-sphere fluid and $\alpha\left(\eta_{c}^{\prime}, q^{\prime}\right)$ is the polymer free-volume fraction, i.e., the fraction of the total volume accessible to the polymer centers (not excluded by the colloids). The free-volume fraction is related to the excess chemical potential of the polymer via Widom's particle insertion $\operatorname{method}^{57}$ :

$$
\mu_{p, \text { ex }}=-k_{\mathrm{B}} T \ln \left\langle\exp \left(-\Delta U / k_{\mathrm{B}} T\right)\right\rangle=-k_{\mathrm{B}} T \ln \alpha,
$$

where the angular brackets denote an ensemble average over colloid configurations and polymer positions, and $\Delta U$ is the change in potential energy upon insertion of a polymer here infinite if the polymer overlaps a colloid and zero otherwise. An approximation for $\alpha$ is obtained by noting that $\mu_{p \text {,ex }}$ also equals the reversible work required to produce a spherical cavity, of radius $R_{p}^{\prime}$, in a fluid of hard sheres of radius $R_{c}^{\prime}$. The scaled-particle approximation 58.59 for $\mu_{p, \text { ex }}$ then yields

$$
\alpha\left(\eta_{c}^{\prime}, q^{\prime}\right)=\left(1-\eta_{c}^{\prime}\right) \exp \left(-A x-B x^{2}-C x^{3}\right)
$$

with $x=\eta_{c}^{\prime} /\left(1-\eta_{c}^{\prime}\right), A=q^{\prime 3}+3 q^{\prime 2}+3 q^{\prime}, B=3 q^{\prime 3}+9 q^{\prime 2} / 2$, and $C=3 q^{\prime 3}$. Within the same approximation, the excess free energy of the colloids is given by

$$
\beta \phi_{\mathrm{HS}}\left(\eta_{c}^{\prime}\right)=\frac{3 \eta_{c}^{\prime}\left[3 \eta_{c}^{\prime}\left(2-\eta_{c}^{\prime}\right)-2\left(1-\eta_{c}^{\prime}\right)^{2} \ln \left(1-\eta_{c}^{\prime}\right)\right]}{8 \pi R_{c}^{\prime 3}\left(1-\eta_{c}^{\prime}\right)^{2}}
$$


The pressure resulting from Eq. (15) coincides with the compressibility equation of state following from the exact solution of the Percus-Yevick integral equation for hard spheres ${ }^{60.61}$. Taken together, Eqs. (11)-(15) provide an approximation for the fluid free energy, from which the fluid-fluid demixing phase diagram can be computed.

\section{RESULTS AND DISCUSSION}

Based on the approximate free energy of Eqs. (111)-(15), we have performed a coexistence analysis to determine the equilibrium fluid-fluid demixing binodal, defined by equality of pressures and chemical potentials in coexisting colloid-rich and colloid-poor fluid phases. We restrict our study to parameters for which crystallization occurs only at higher colloid densities, well separated from fluid-fluid demixing. Figure 3 presents the resulting phase diagrams for a fixed polymer-to-colloid size ratio, $q=1$, and for effective polymer-to-colloid size ratios $q^{\prime}=1,0.8,0.6,0.4$, corresponding to electrostatic colloid size ratios $\xi=1,1.11,1.25$, 1.43, i.e., varying ionic strengths [varying $\kappa$ in Eq. (II)]. We assume, as discussed in Sec. III] that $\xi$ is independent of colloid density, which is valid in the high-salt-concentration regime $\left(\rho_{s} \gg Z \rho_{c}\right)$, where salt ions overwhelm counterions and $\kappa$ [Eq. (2)] is essentially independent of $\rho_{c}$. In the extreme limit, $\kappa \rightarrow \infty(\xi \rightarrow 1)$, we recover the binodal for the purely entropic case of neutral colloids. With decreasing ionic strength (decreasing $\kappa$, increasing effective colloid radius), the binodal shifts to significantly higher polymer concentrations and the critical point to slighty lower colloid concentrations. The qualitative consequence of lowering ionic strength is thus a significant enhancement of stability against fluid-fluid demixing. In the absence of polymer $\left(\eta_{p}=0\right)$, the mixture reduces to a one-component system of hard spheres of effective radius $R_{c}^{\prime}$. In this limit, crystallization occurs at effective colloid volume fraction $\eta_{c}^{\prime}=\xi^{3} \eta_{c}=0.494$, which is well above the demixing critical volume fractions for $\xi$ values considered here (see Fig. (3). Note that the case $\xi=1.43$ is close to the limit beyond which demixing becomes metastable with respect to the freezing transition. At higher values of $\xi$, freezing may preempt demixing - a scenario that we do not explore here.

The enhanced stability of charged-colloid - neutral-polymer mixtures can be physically interpreted in terms of a weakening of polymer depletion-induced colloidal attraction. Electrostatic repulsion increases the average separation between macroions, tending to lower the 
frequency of configurations in which polymer is depleted from the intervening space. In our model, as the range of electrostatic repulsion increases (e.g., by removing salt), the effective polymer-to-colloid size ratio decreases, reducing the range of the effective colloid-colloid attraction, and thus diminishing the driving force for phase separation.

A physically equivalent interpretation of the electrostatic suppression of demixing follows from considering the polymer free-volume fraction $\alpha$. From inspection of Eq. (14), it is not immediately obvious how $\alpha$ varies with electrostatic colloid size ratio $\xi$, given that, with increasing $\xi$, the quantities $\left(1-\eta_{c}^{\prime}\right), A, B$, and $C$ all decrease, while $x$ increases. As Fig. 4 illustrates, however, with increasing electrostatic repulsion between colloids, the free volume available to the polymer coils is monotonically reduced, even though the colloid-polymer interaction is unchanged. The reason for this reduction is that more strongly repelling colloids remain more widely separated, allowing less excluded volume to be hidden within overlapping polymer depletion shells surrounding the colloids. The lower free-volume fraction reduces the polymer entropy in the colloid-rich phase, thereby suppressing demixing.

As a check on the consistency of the above interpretations, we consider the liquid-vapor interfacial tension $\gamma$ as a function of the colloid volume fraction difference $\Delta \eta_{c}$ between the coexisting phases. To calculate $\gamma$, we exploit previous density-functional (DF) theory results ${ }^{19,62}$ for the AO model, which have been found to be in reasonable agreement with simulation ${ }^{23.24}$. By appropriately rescaling the DF predictions of Ref. $19\left(\Delta \eta_{c} \rightarrow \Delta \eta_{c} / \xi^{3}\right.$ and $\beta \gamma \sigma_{c}^{2} \rightarrow \beta \gamma \sigma_{c}^{2} / \xi^{2}$, we obtain the results shown in Fig. 5. Evidently, the interfacial tension increases with increasing effective colloid radius, which is consistent with the predicted suppression of liquid-vapor demixing. Although the mapping of DF theory from neutral to charged systems is straightforward in the absence of external potentials, more care may be required in the presence of a surface. For example, a hard wall for the neutral system becomes a non-additive wall for the charged system. In such cases, coupling to the external potential may have to be explicitly built into the theory.

\section{SUMMARY AND CONCLUSIONS}

In summary, we have investigated fluid-fluid demixing in mixtures of charged colloids and neutral non-adsorbing polymers. Our theoretical approach involves first mapping a model 
mixture of charged hard-sphere colloids - interacting via an effective Yukawa electrostatic pair potential - and ideal polymer spheres onto an effective Asakura-Oosawa model with purely excluded-volume interactions, and then applying free-volume theory and densityfunctional theory to study, respectively, bulk phase separation and interfacial tension. The effective model is characterized by a single dimensionless parameter, namely the ratio of the polymer diameter to the effective colloid diameter.

Within this simple framework, we find that increasing the range of the electrostatic repulsion between macroions (e.g., by decreasing salt concentration) suppresses demixing and increases the interfacial tension between coexisting colloid-rich and colloid-poor phases. Hence, our main conclusion is that electrostatic interactions stabilize the suspension against depletion-induced phase separation. As the depletion-induced attraction is short-ranged, the principal mechanism is analogous to charge-stabilization against coagulation induced by van der Waals forces. In both cases the electrostatic repulsion keeps the colloidal particles (at least partially) outside the range of attraction. Within our free-volume approach, the effect is clearly manifested through a reduction of the effective polymer size, and a correspondingly weaker depletion-induced attraction. The predicted increase of miscibility with decreasing salt concentration is qualitatively consistent with the observed behavior of mixtures of charged proteins and uncharged non-adsorbing polysaccharides (e.g., gelatin and dextran), which are constituents of many food colloids $\frac{63}{}$. Future experiments and simulations would help to further test the qualitative trends predicted here.

A possible extension of the present theory, which could yield more quantitative predictions, would be based on a variational method for the free energy. This method would split the free energy into two parts: a (zeroth order) reference term, describing the effective AO model, and a (first-order) perturbative term, describing the repulsive tail of the colloidal pair potential. Minimizing the total free energy with respect to the effective colloid hard-sphere diameter would give an upper bound on the true free energy. Such an approach might be better suited to describing colloid-polymer mixtures with long-range electrostatic repulsion between the colloids, which have been studied in recent experiments ${ }^{64,65}$, and could be applied also to mixtures of colloids and nonideal polymers, e.g., polyelectrolytes ${ }^{40,41.42 .43,44}$, properly incorporating soft polymer-polymer interactions ${ }^{29}$.

In this paper, we have focused attention on bulk fluid-fluid demixing in the high-salt- 
concentration regime. As an outlook for future work, we mention two related phenomena that could be addressed within a similar theoretical framework. First, the fluid-solid (freezing) phase behavior of colloid-polymer mixtures is likely to be enriched by combining electrostatic

and polymer-depletion interactions. It is well known ${ }^{1.66}$ that concentrated suspensions of charged colloids crystallize into close-packed fcc or hcp structures at higher ionic strengths, and into the more open bcc structure at lower ionic strengths. It would be interesting to examine the competing influences of electrostatics and polymer depletion on freezing and the relative stabilities of crystal structures.

Second, strongly deionized suspensions of highly charged colloids are known to exhibit surprisingly complex phase behavior. For example, experimental observations 67.68 and theoretical predictions ${ }^{48,50,52}$ indicate the possibility of unusual bulk separation into macroionrich and -poor phases, driven by a competition between counterion entropy and macroioncounterion attractive energy. Incorporating the volume energy [Eq. (3)] into the total free energy would make possible an exploration of the influence of added depletants on counterionfacilitated phase separation in deionized suspensions.

\section{Acknowledgments}

This work was supported by the National Science Foundation under Grant No. DMR0204020 and by the SFB TR6 of the DFG. Helpful discussions with Moreno Fasolo and Remco Tuinier are gratefully acknowledged. 
1 P. N. Pusey, in Liquids, Freezing and Glass Transition, session 51, ed. J.-P. Hansen, D. Levesque, and J. Zinn-Justin (North-Holland, Amsterdam, 1991).

2 W. C. K. Poon, J. Phys.: Condens. Matter 14, R859 (2002).

3 R. Tuinier, J. Rieger, and C. G. de Kruif, Adv. Colloid Interface Sci. 103, 1 (2003).

4 D. G. A. L. Aarts, J. H. van der Wiel, H. N. W. Lekkerkerker, J. Phys.: Condens. Matter 15, S245 (2003).

5 D. G. A. L. Aarts, M. Schmidt, and H. N. W. Lekkerkerker, Science 304, 847 (2004).

6 D. G. A. L. Aarts and H. N. W. Lekkerkerker, J. Phys.: Condens. Matter 16, S4231 (2004).

7 A colloidal quasicrystal phase has been predicted [A. R. Denton and H. Löwen, Phys. Rev. Lett. 81, 469 (1998)], but not yet conclusively observed.

8 S. Asakura and F. Oosawa, J. Chem. Phys. 22, 1255 (1954).

9 A. Vrij, Pure and Appl. Chem. 48, 471 (1976).

10 A. P. Gast, C. K. Hall, and W. B. Russel, J. Coll. Int. Sci. 96, 251 (1983).

11 H. N. W. Lekkerkerker, W. C. K. Poon, P. N. Pusey, A. Stroobants, and P. B. Warren, Europhys. Lett. 20, 559 (1992).

12 D. G. A. L. Aarts, R. Tuinier, and H. N. W. Lekkerkerker, J. Phys.: Condens. Matter 14, 7551 (2002).

13 M. Schmidt, H. Löwen, J. M. Brader, and R. Evans, Phys. Rev. Lett. 85, 1934 (2000).

14 M. Dijkstra, J. M. Brader, and R. Evans, J. Phys.: Condens. Matter 11, 10079 (1999).

15 P. G. Bolhuis, A. A. Louis, and J.-P. Hansen, Phys. Rev. Lett. 89, 128302 (2002).

16 R. L. C. Vink, J. Horbach, and K. Binder, Phys. Rev. E 71, 011401 (2005).

17 A. Vrij, Physica A 235, 120 (1997).

18 J. M. Brader and R. Evans, Europhys. Lett. 49, 678 (2000).

19 J. M. Brader, R. Evans, M. Schmidt, and H. Löwen, J. Phys.: Condens. Matter 14, L1 (2002).

20 A. A. Louis, P. G. Bolhuis, E. J. Meijer, and J.-P. Hansen, J. Chem. Phys. 116, 10547 (2002).

21 A. Moncho-Jorda, B. Rotenberg, A. A. Louis, J. Chem. Phys. 119, 12667 (2003).

22 D. G. A. L. Aarts, R. P. A. Dullens, H. N. W. Lekkerkerker, D. Bonn, and R. van Roij, J. Chem. Phys. 120, 1973 (2004). 
23 R. L. C. Vink and J. Horbach, J. Chem. Phys. 121, 3253 (2004).

24 R. L. C. Vink and J. Horbach, J. Phys.: Condens. Matter 16, S3807 (2004).

25 A. Moncho-Jordá, J. Dzubiella, J.-P. Hansen, and A. A. Louis, cond-mat/0411282, to appear in J. Phys. Chem. B (2005).

26 R. L. C. Vink and M. Schmidt, to appear in Phys. Rev. E.

27 E. J. Meijer and D. Frenkel, Phys. Rev. Lett. 67, 1110 (1991); J. Chem. Phys. 100, 6873 (1994); Physica A 213, 130 (1995).

28 R. Dickman and A. Yethiraj, J. Chem. Phys. 100, 4683 (1994).

29 A. A. Louis, P. G. Bolhuis, J.-P. Hansen, and E. J. Meijer, Phys. Rev. Lett. 85, 2522 (2000).

30 M. Fuchs and K. S. Schweizer, Europhys. Lett. 51, 621 (2000); Phys. Rev. E 64, 021514 (2001);

J. Phys.: Condens. Matter 14, R239 (2002).

31 M. Schmidt and A. R. Denton, Phys. Rev. E 65, 021508 (2002).

32 M. Schmidt and A. R. Denton, Phys. Rev. E 65, 061410 (2002).

33 M. Schmidt, A. R. Denton, and J. M. Brader, J. Chem. Phys. 118, 1541 (2003).

34 A. R. Denton and M. Schmidt, J. Phys.: Condens. Matter 14, 12051 (2002).

35 M. Fasolo and P. Sollich, cond-mat/0410374 (2004).

36 R. P. Sear and D. Frenkel, Phys. Rev. E 55, 1677 (1997).

37 M. Fasolo and P. Sollich, J. Phys.: Condens. Matter 17, 797 (2005).

38 P. R. Sperry, J. Coll. Int. Sci. 99, 97 (1984).

39 A. P. Gast, W. B. Russel, and C. K. Hall, J. Coll. Int. Sci. 109, 161 (1986).

40 O. Mondain-Monval, F. Leal-Calderon, J. Phillip, and J. Bibette, Phys. Rev. Lett. 75, 3364 (1995).

41 M. Piech and J. Y. Walz, J. Coll. Int. Sci. 253, 117 (2002); A. Sharma, S. N. Tan, and J. Y. Walz, J. Coll. Int. Sci. 191, 236 (1997).

42 G. Petekidis, L. A. Galloway, S. U. Egelhaaf, M. E. Cates, and W. C. K. Poon, Langmuir 18, 4248 (2002).

43 L. Helden, G. H. Koenderink, P. Leiderer, and C. Bechinger, Langmuir 14, 5662 (2004).

44 P. G. Ferreira, M. Dymitrowska, and L. Belloni, J. Chem. Phys. 113, 9849 (2000).

45 J.-P. Hansen and I. R. McDonald, Theory of Simple Liquids, $2^{\text {nd }}$ ed. (Academic, London, 1986). 
B. V. Derjaguin and L. Landau, Acta Physicochimica (USSR) 14, 633 (1941); E. J. W. Verwey and J. T. G. Overbeek, Theory of the Stability of Lyophobic Colloids (Elsevier, Amsterdam, 1948).

47 M. J. Grimson and M. Silbert, Mol. Phys. 74, 397 (1991).

48 R. van Roij and J.-P. Hansen, Phys. Rev. Lett. 79, 3082 (1997).

49 H. Graf and H. Löwen, Phys. Rev. E 57, 5744 (1998).

50 R. van Roij, M. Dijkstra, and J.-P. Hansen, Phys. Rev. E 59, 2010 (1999).

51 A. R. Denton, J. Phys.: Condens. Matter 11, 10061 (1999); Phys. Rev. E 62, 3855 (2000).

52 P. B. Warren, J. Chem. Phys. 112, 4683 (2000).

53 P.-G. de Gennes, Scaling Concepts in Polymer Physics (Cornell, Ithaca, 1979).

54 J. A. Barker and D. Henderson, J. Chem. Phys. 47, 4714 (1967).

55 R. Tuinier, private communication.

56 H. C. Andersen, J. D. Weeks, and D. Chandler, Phys. Rev. A 4, 1597 (1971); J. D. Weeks, D. Chandler, and H. C. Andersen, J. Chem. Phys. 54, 5237 (1971).

57 B. Widom J. Chem. Phys. 39, 2808 (1963).

58 H. Reiss, H. L. Frisch, and J. L. Lebowitz, J. Chem. Phys. 31, 369 (1959).

59 J. L. Lebowitz, E. Helfand, and E. Praestgaard J. Chem. Phys. 43, 774 (1965).

60 M. Wertheim, Phys. Rev. Lett. 8, 321 (1963).

61 E. Thiele, J. Chem. Phys. 38, 1959 (1963).

62 P. P. F. Wessels, M. Schmidt, and H. Löwen, J. Phys.: Condens. Matter 16, L1 (2004).

63 V. B. Tolstoguzov, Food Hydrocolloids 4, 429 (1991).

64 A. Stradner, H. Sedgwick, F. Cardinaux, W. C. K. Poon, S. U. Egelhaaf, and P. Schurtenberger, Nature 432, 492 (2004).

65 A. I. Campbell, V. J. Anderson, J. S. van Duijneveldt, and P. Bartlett, cond-mat/0412108 (2004).

66 Y. Monovoukas and A. P. Gast, J. Coll. Int. Sci. 128, 533 (1989).

67 B. V. R. Tata, M. Rajalakshmi, and A. K. Arora, Phys. Rev. Lett. 69, 3778 (1992).

68 K. Ito, H. Yoshida, and N. Ise, Science 263, 66 (1994). 


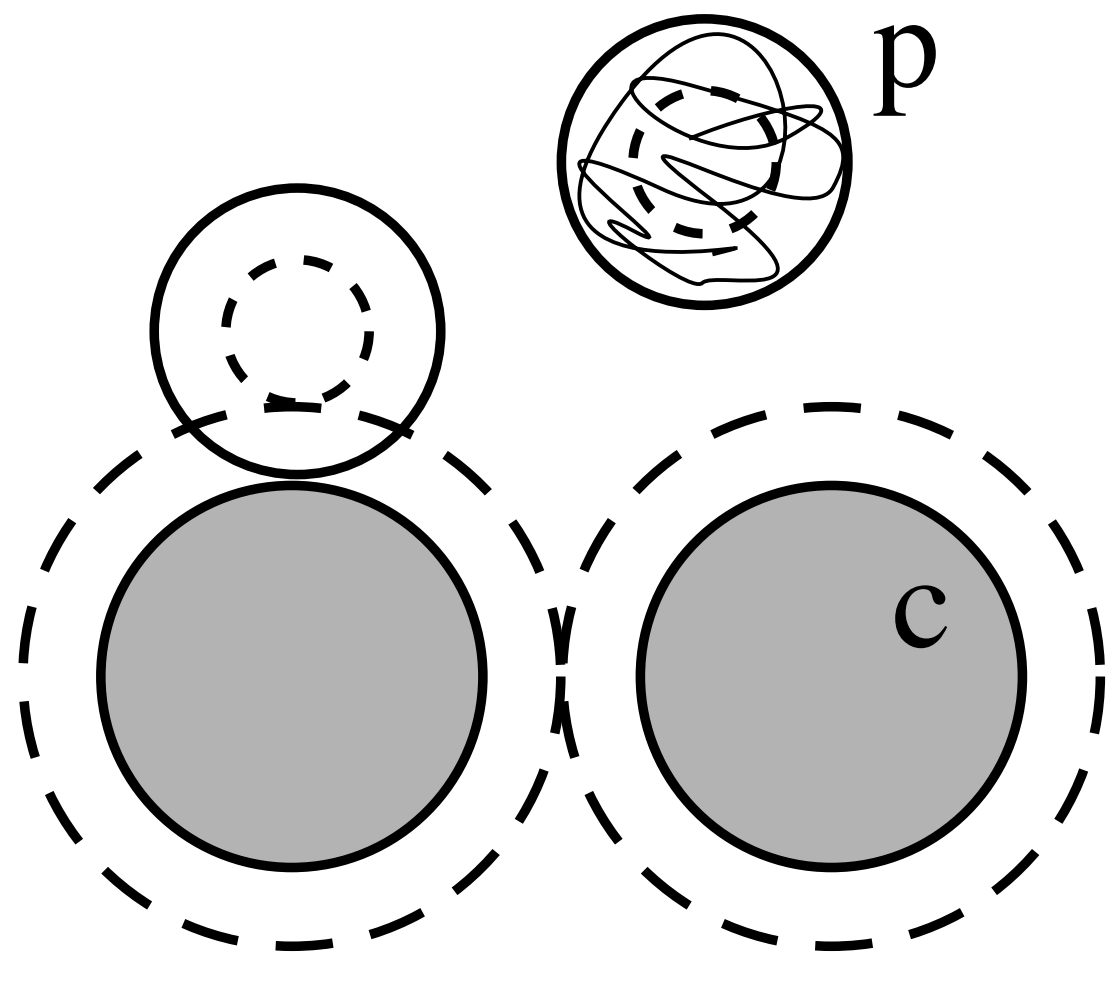

FIG. 1: Sketch of the two models of charged colloids (c) and (effective spherical) neutral polymer (p). The solid curves indicate the actual sizes of the particles (radii $R_{c}$ and $R_{p}$ ) in the original model, and the dashed curves the rescaled sizes (radii $R_{c}^{\prime}$ and $R_{p}^{\prime}$ ) in the effective Asakura-Oosawa model. Note that the depletion layer thickness is the same in both models. 

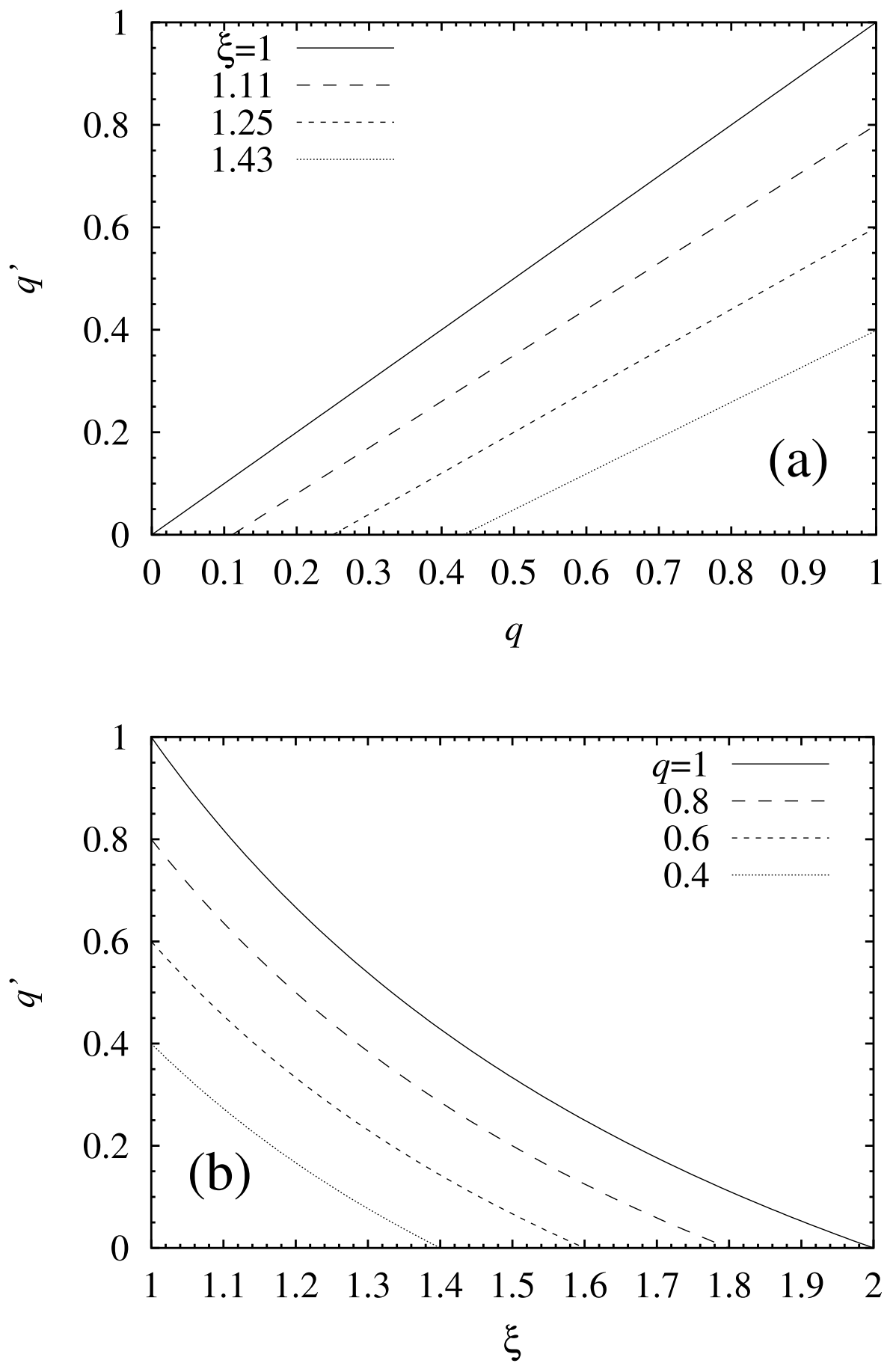

FIG. 2: Effective polymer-to-colloid size ratio $q^{\prime}=R_{p}^{\prime} / R_{c}^{\prime}$ vs. (a) actual polymer-to-colloid size ratio $q=R_{p} / R_{c}$ and (b) electrostatic colloid size ratio $\xi=R_{c}^{\prime} / R_{c}$. 


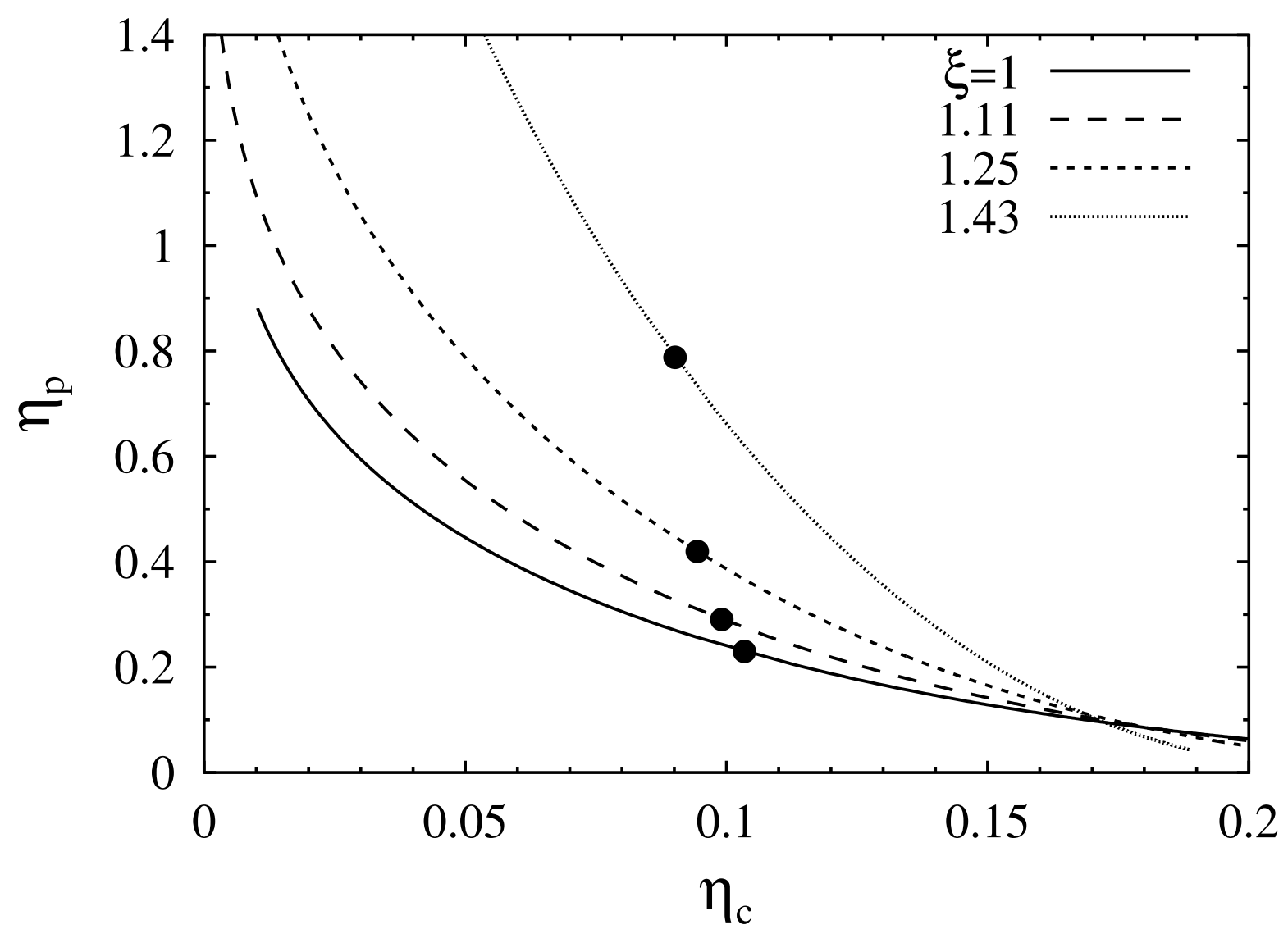

FIG. 3: Fluid-fluid demixing binodal of charged-colloid - neutral-polymer mixtures as a function of colloid and polymer volume fractions, $\eta_{c}$ and $\eta_{p}$, for fixed polymer-to-colloid size ratio, $q=R_{p} / R_{c}=$ 1 , and varying electrostatic colloid size ratio $\xi=R_{c}^{\prime} / R_{c}$. From bottom to top, $\xi=1,1.11,1.25,1.43$, corresponding to increasing effective colloid radius. Symbols represent the respective critical points. 


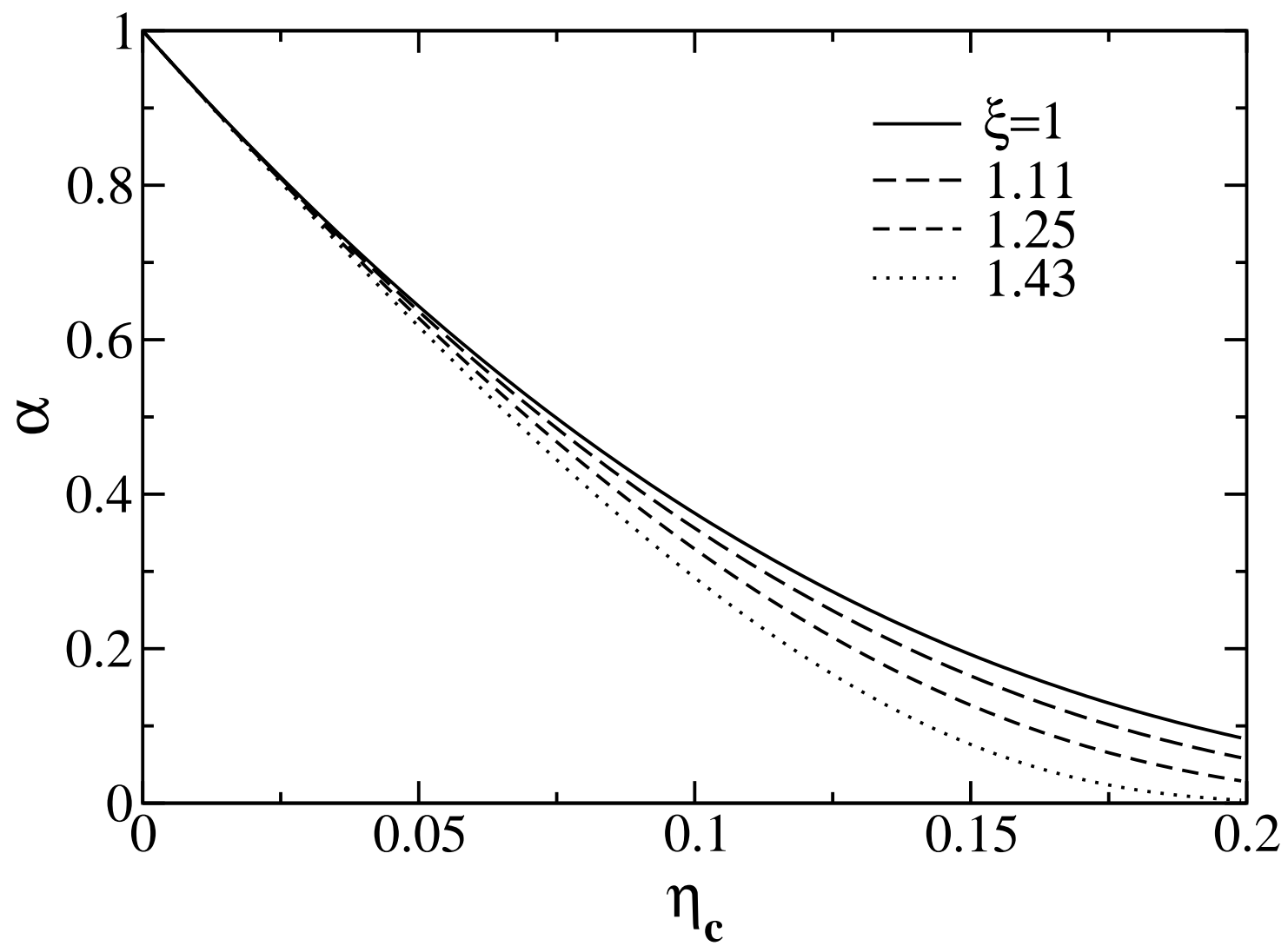

FIG. 4: Polymer free-volume fraction $\alpha$ [from Eq. (14)] as a function of colloid volume fraction $\eta_{c}$ for fixed polymer-to-colloid size ratio, $q=R_{p} / R_{c}=1$, and varying electrostatic colloid size ratio $\xi=R_{c}^{\prime} / R_{c}$. From top to bottom, $\xi=1,1.11,1.25,1.43$, corresponding to increasing effective colloid radius. 


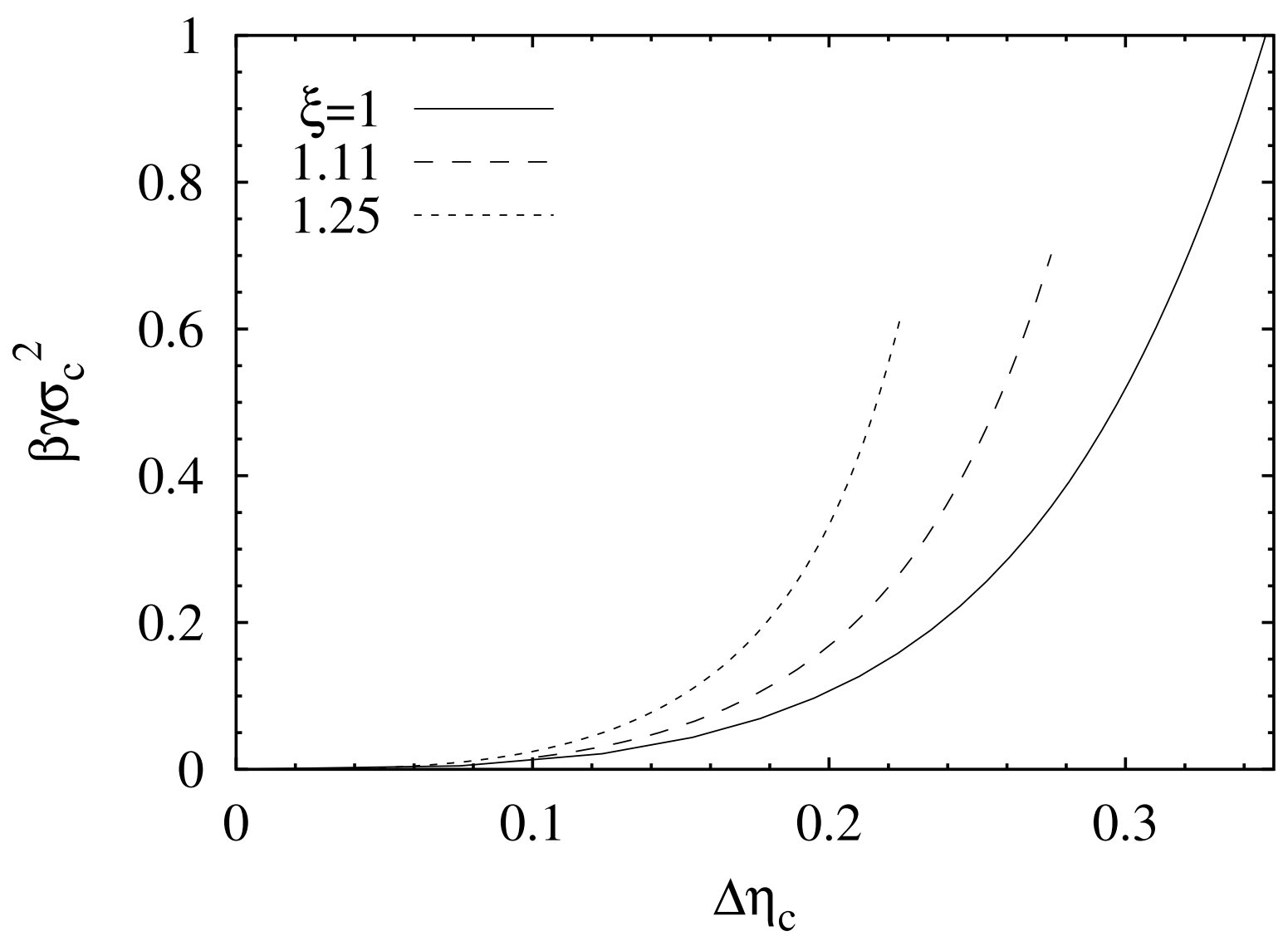

FIG. 5: Interfacial tension $\gamma$, in reduced units, between liquid (colloid-rich) and vapor (colloidpoor) phases vs. the difference in colloid volume fraction $\Delta \eta_{c}$ between colloidal liquid and vapor (at bulk coexistence). 